\title{
Research biobanks: A two-faced future
}

AUTHORS:

Marco Capocasa' ${ }^{1}$ ID
Valentina Dominici
Fabrizio Rufo ${ }^{1,2}$
AFFILIATIONS:
IItalian Institute of Anthropology,
Rome, Italy
${ }^{2}$ Department of Environmental
Biology, Sapienza University of
Rome, Rome, Italy

\section{CORRESPONDENCE TO:}

Marco Capocasa

\section{EMAIL:}

marco.capocasa@yahoo.it

\section{KEYWORDS:}

biobanking; accessibility; biological samples; data; network

\section{HOW TO CITE:}

Capocasa M, Dominici V, Rufo F. Research biobanks: A two-faced future. S Afr J Sci. 2018;114(11/12), Art. \#5402, 3 pages. https://dx.doi. org/10.17159/sajs.2018/5402

\section{PUBLISHED:}

27 November 2018
The possibility of preserving human tissue separated from the body, from which to extract clinical information, even on large numbers of individuals with similar clinical conditions, represents a great opportunity for the progress of biomedicine. To date, it is virtually impossible to hypothesise all the future uses of such tissue. However, the decisive role of these biological materials in the understanding and resolution of questions regarding the origin and development of certain genetic diseases is well recognised.

The progress of genetic and biotechnological research has led to the proliferation of collections of biological materials by institutions called 'biobanks': repositories that store human biological samples, with or without linking them to genetic or clinical data. ${ }^{1}$ Usually biobanks are part of large public research centres, small hospitals and pharmaceutical companies. They conduct their activities following different, and not always standardised, storage and conservation protocols. Because of this enormous heterogeneity of structures, materials and methodologies, research biobanks have been subjected to profound ambiguity and fragmentation, as well as uncertainty in terms of regulation. This situation has encouraged discussion on the legal rules regarding their activities, particularly for the protection of donors' rights. ${ }^{2-4}$

In the 21 st century, the technological progress in automation and archival informatisation and the development of the World Wide Web boosted a radical revolution of biobanking. Marked changes have occurred in the context of the management of biological samples, particularly in regard to their transport and storage. However, progress has also been made in regard to the ethical and legal requirements necessary to ensure the privacy and safety of donors and the long-term availability of these materials. Collecting samples in a more accurate and ethical way may be a crucial contribution in the advancement of clinical and biomedical trials and, more generally, in the creation of more informative data sets. Such progress is also associated with a substantial increase in the production of data as a consequence of the collection of pathological, epidemiological, environmental and ethno-social information of the donors.

The collection of biological materials in compliance with ethical and legal standards could be seen as the main innovation of research biobanks. Firstly, collections of samples are virtually unusable today because of the lack of detail in the informed consent or because they were catalogued following inappropriate methods. ${ }^{5}$ Overcoming these issues represents one of the main enhancements for the development of modern and more structured biobanks.

\section{Biobanking: Between politics and economics}

Research biobanks often operate in very different contexts and, in many cases, their activities have become of primary interest to government agencies. In fact, the relationship between biobanks and politics is strengthening as many governments are increasingly interested in the efficiency and standardisation of ethical-legal frameworks for the sampling of biological materials. There is reciprocity between scientists and politicians: the collection of more, and extensively useable, human biological samples helps the former to conduct more accurate studies and guarantees the support of the latter regarding their political choices on scientific research.

Biobanking has also revealed unprecedented business opportunities. In 2011, Global Industry Analysts released a global report on the biobank market, in which they predicted that the market in 2017 for high-quality human biological samples would be USD22.3 billion. Thus, the political and economic interests of biobanking are clear.

Scientific interests are obviously the first priority. There is no doubt that, globally, the development and growth of biobanks has exponentially increased opportunities for analysing and studying the collection of human biological samples and data extrapolated therefrom. This growth corresponds to an increase in research perspectives compared with those possible if the sampling and management of biological materials was still exclusively tied to the initiatives of individual research groups. ${ }^{1}$ In addition to these new opportunities, the rapid evolution of biobanking has also created new challenges and obstacles. Particularly, researchers have difficulty in accessing biobank resources. The propensity of these institutions to share their samples and data with the scientific community is a controversial subject which encompasses a double challenge: for researchers, gaining access to biological samples and data, and, for biobanks, finding a balance between the scientific interests of researchers and donors' expectations. A first step towards a solution capable of satisfying both sides could be represented by a better and more flexible use of current forms of informed consent. ${ }^{6,7}$ However, the requirement of a consent form including a section ensuring the sustainability of a wide accessibility to samples and data does not solve the problem related to economic interests, which are often hidden and protected by the scientific aim itself.

Although biobanks have suddenly become more 'open', several bioethical issues related to the sharing procedures of biological resources have emerged. Starting from the drafting of informed consent, biobanks must take into account a number of 'twists' in the definition of the section concerning the handling of samples and data. They should meet the requirements of ethical committees. They should also clearly state the hypothetical future uses of their resources. Moreover, they should enable potential donors to make truly informed decisions.

Accessibility to biobank resources is generally conditional on the fulfilment of specific, often very compelling, requirements and seems to be related to three main aspects. ${ }^{8}$ The first aspect is the transparency between biobanks and applicants. Particularly, before allowing others to use their own materials, biobanks want to know the scientific aims of applicants. This request is strictly linked with specific sharing statements reported in the original consent form. It also provides a certain level of control by the biobanks regarding the scientific reliability and reputation (c) 2018. The Author(s). Published under a Creative Commons Attribution Licence. 
of applicants and their research groups, in order to reduce the risk of misuse of biobank resources. This is an ethical and technical approach to the management of scientific resources that can foster public trust in the work of these institutions, thus increasing willingness to participate in their activities.

Secondly, accessibility is related to the availability and origin of research funds. Biobanks seem to be more prone to collaborate with research groups that are publicly funded. This trend reflects the Organisation for Economic Co-operation and Development (OECD)'s recommendation in 2007 for open access to scientific resources, in which these resources are defined as 'public goods'. The OECD considers the sharing of these resources to be a means of enhancing public investment in scientific research. The availability of funds is a criterion adopted by biobanks in deciding whether or not to provide their resources to third parties. The presence of clauses directly linked to certain economic benefits for biobanks reveals their possible 'second nature' as institutions which also make a profit in providing bio-collection and storage services. However, it is not clear if this commercial nature operates as a sharing barrier, thereby lowering the risk of exploitation of their resources. As suggested by Caulfield and colleagues ${ }^{9}$, sample and data sharing is a practice that can be influenced, or even hindered, by the introduction of private funding and by collaboration with private groups. In fact, the latter could have economic expectations concerning the use of resources produced with their money. Consequently, these groups could have entered into agreements governing such collaborations by acting as sharing barriers.

Thirdly, accessibility is related to co-authorship. Some biobanks require recognition as co-authors on publications resulting from research based on the analysis of their samples and data. Other authors have highlighted this typical bad practice in the sharing behaviour of research groups. ${ }^{10-12}$ Clearly, it contributes to the spread of a climate of mistrust and a lower propensity for cooperation within the scientific community.

These findings suggest that both economic and academic aspects are involved in determining ways to manage the exploitation of biological resources by biobanks currently operating on a global scale. However, biobanks can differ completely in goals and outcomes and diametrically opposed visions can coexist within the same biobank. We should also take into account that biobanks are regulated on the basis of both national and international rules (such as the Transatlantic Trade and Investment Partnership bilateral agreement between the USA and the European Union), which sometimes hinder harmonisation, with consequences that may affect national health services and, more generally, biomedical research.

\section{Looking to tomorrow}

The availability of high-quality samples, accompanied by detailed metadata, will be the decisive push for the discovery of previously unknown biomarkers, thus facilitating the definition of new and innovative therapies. ${ }^{13,14}$ The only way to increase the number and variety of human biological samples is to increase the 'source of tissues' and the efficiency of their provision. ${ }^{5}$ The future of biobanks will depend, first and foremost, on their ability to respond to this increased demand. However, biobanks will only be able to overcome this challenge if they can also significantly reduce sampling and distribution costs. If we look at biobanking from this point of view, its tomorrow will be a matter of business in which only those institutions that will be able to operate efficiently and sustainably will remain and compete.

In this scenario, biobank networks can be seen as the most promising strategy to try to facilitate accessibility to samples and their findability. In the USA, the Cooperative Human Tissue Network (CHTN) is a 'generalised biobank' capable of collecting any kind of human samples for any biomedical research. In Europe, the Biobanking and BioMolecular Resources Research Infrastructure (BBMRI-ERIC) has been in operation since 2013 and is probably the world's largest biobank network. BBMRI-ERIC provides access to human biological samples that are considered raw materials needed for the advancement and development of biomedicine in Europe. Consortia such as CHTN and BBMRI-ERIC are increasingly essential, particularly for those research groups that do not have enough funding and human capacity to maintain an efficient biobank. However, while these networks are organised and equipped with impressive numbers of high-quality samples, obviously they will not actually meet all the needs of research groups. This is because certain studies require a specific type of biological material and a large number of samples collected from a specific population. Thus, there will still be room for local and small biobanks.

In the near future, another aspect that will affect biobanks' choices in collecting samples, particularly regarding the type of tissue and the sample size, will be the so-called 'post-genomic revolution'. It will force researchers around the world towards increasingly specific sample requirements that will reverberate in the activity of biobanks, particularly in the need to develop more coordinated collections to satisfy specific scientific issues. De Souza and Greenspan ${ }^{15}$ point out that this process has already begun, as evidenced by the advent of population biobanks, as well as biobanks which collect only DNA or focus their activity on a single pathology.

We can conclude that the future of biobanking is double-edged. Bright and, at the same time, full of obstacles. First of all, its evolution will depend on addressing some of the unsolved problems preventing the full exploitation of resources, mostly in regard to the lack of shared standards for collecting, cataloguing and managing samples. Another issue is the long-term sustainability of these institutions. The scientific community needs efficient biobanks to support long-term studies. However, this aspect would require a separate study of the critical points linked with the maintenance of such institutions and the efficiency of their activities in supporting actively and dynamically the research enterprise. Biobanks will also have to face the question of open access to their resources. As discussed above, there are still barriers to the sharing of samples and data between biobanks and researchers. The fact that the scientific community is fully aware of the importance of sharing does not necessarily mean that these barriers are harmful and unfair and that they should not exist. In fact, some of them play an important role, because they exist to guarantee the fundamental rights of donors and prevent the misuse of biological materials. Thus, respect for donors' rights should always be given consideration in trying to overcome these barriers, not as a means to unify all the procedures for accessing biological resources but to find a definition of a globally recognised operating standard.

\section{Acknowledgements}

This work was supported by the Istituto Italiano di Antropologia (www.isita-org.com).

\section{References}

1. Haga SB, Beskow LM. Ethical, legal, and social implications of biobanks for genetics research. Adv Genet. 2008;60:505-544. https://doi.org/10.1016/ S0065-2660(07)00418-X

2. Novelli G, Pietrangeli I. I campioni biologici [Biological samples]. In: Rodotà S, Tallacchini M, editors. Trattato di biodiritto [Treatise of biolaw]. Milan: Giuffrè; 2010. p. 1027-1061. Italian.

3. Hoeyer KL. Size matters: The ethical, legal, and social issues surrounding largescale genetic biobank initiatives. Nor Epidemiol. 2012;21:211-220. https://doi. org/10.5324/nje.v21i2.1496

4. Macilotti M. Informed consent and research biobanks: A challenge in three dimensions. In: Pascuzzi G, Izzo U, Macilotti M, editors. Comparative issues in the governance of research biobanks. Berlin: Springer; 2013. p. 143-161. https://doi.org/10.1007/978-3-642-33116-9_9

5. Somiari SB, Somiari RI. The future of biobanking: A conceptual look at how biobanks can respond to the growing human biospecimen needs of researchers. Adv Exp Med Biol. 2015;864:11-27. https://doi.org/10.1007/9783-319-20579-3_2

6. Kaye J. The tension between data sharing and the protection of privacy in genomics research. Annu Rev Genomics Hum Genet. 2012;13:415-431. https://doi.org/10.1146/annurev-genom-082410-101454

7. D'Abramo F. Biobank research, informed consent and society. Towards a new alliance? J Epidemiol Community Health. 2015;69:1125-1128. https://doi. org/10.1136/jech-2014-205215 
8. Capocasa M, Anagnostou P, D’Abramo F, Matteucci G, Dominici V, Destro Bisol G, et al. Samples and data accessibility in research biobanks: An explorative survey. PeerJ. 2016;4, e1613, 18 pages. https://doi.org/10.7717/ peerj. 1613

9. Caulfield T, Burningham S, Joly Y, Master Z, Shabani M, Borry P, et al. A review of the key issues associated with the commercialization of biobanks. J Law Biosci. 2014;1:94-110. https://doi.org/10.1093/jlb/lst004

10. Vogeli C, Yucel R, Bendavid E, Jones LM, Anderson MS, Louis KS, et al. Data withholding and the next generation of scientists: Results of a national survey. Acad Med. 2006;81:128-136. https://doi.org/10.1097/00001888200602000-00007

11. Milanovic F, Pontille D, Cambon-Thomsen A. Biobanking and data sharing: A plurality of exchange regimes. Genomics Soc Policy. 2007;3:17-30. https:// doi.org/10.1186/1746-5354-3-1-17
12. Tenopir C, Allard S, Douglass K, Aydinoglu AU, Wu L, Read E, et al. Data sharing by scientists: Practices and perceptions. PLoS ONE. 2011;6, e21101, 21 pages. https://doi.org/10.1371/journal.pone.0021101

13. Shaw PM, Patterson SD. The value of banked samples for oncology drug discovery and development. J Natl Cancer Inst Monogr. 2011;42:46-49. https://doi.org/10.1093/jncimonographs/lgr004

14. Olson JE, Bielinski SJ, Ryu E, Winkler EM, Takahashi PY, Pathak J, et al. Biobanks and personalized medicine. Clin Genet. 2014;86:50-55. https://doi. org/10.1111/cge.12370

15. De Souza YG, Greenspan JS. Biobanking past, present and future: Responsibilities and benefits. AIDS. 2013;27:303-312. https://doi.org/10.1097/ QAD.0b013e32835c1244 